\title{
Correlated Wave Packet treatment of Neutrino and Neutral Meson Oscillations
}

\author{
Michael Nauenberg \\ Department of Physics \\ University of California, Santa Cruz,
}

Nov. 1998

\begin{abstract}
A quantum wave packet treatment of neutrino and neutral $\mathrm{K}$ and $\mathrm{B}$ meson oscillations is presented which incorporates the recoil particle in the production process, and includes the effect of the localization and lifetime of the source assumed to be a resonance or unstable particle. This approach removes the ambiguities in the conventional single particle treatment of these oscillations, and elucidates the role of quantum correlations with the recoil particle. A fundamental connection between the stochastic decay time of the source and the space-time coordinates of the correlated final state particles is derived.
\end{abstract}

A proper description of neutral $\mathrm{K}$ and $\mathrm{B}$ meson oscillations [1] [2] and neutrino oscillations [3]- [5] requires that the familiar superposition of states with definite mass be represented by coherent wave packets [6] - [10]. However, the conventional single particle treatment of these oscillations leads to ambiguities which have lead to debates whether the momentum [6] - [8] or the energy [11] - 14] remains unchanged for different mass eigenstates, although the resultant transition probabilities are the same in both cases. Furthermore, such descriptions leave unanswered the fundamental question how the properties of a single particle wave packet are determined by the nature of the production process. In this note we resolve these problems by considering a a wave packet which includes a single recoil particle produced by the 
decay of a resonance or unstable particle, and incorporates both energy and momentum conservation [15] -18] instead of resorting to conventional ad-hoc assumptions. Including final state correlations raises some interesting new questions because these correlations take place between space-like separated events, a subtle problem in quantum mechanics discussed a long time ago by Einstein, Podolsky and Rosen (EPR) [19], and considered in connection with the production of a neutral $K \bar{K}$ or $B \bar{B}$ pair in 20] - 22]. In this case oscillations can be observed as EPR correlations between neutral mesons of fixed flavor or their decay products. It has been claimed in [23] - 26] that oscillations of the recoil particle can also be observed even if this particle has a fixed mass. In the case of neutrinos produced in the decay $\pi \rightarrow \mu+\nu$ where the recoil is a charged lepton, this would greatly facilitate experiments. However, Lowe et al. [27]-28] have argued that only a certain traveling pattern of oscillations in the recoil particle coordinates is observable, while Dolgov et al. 18] concluded that such oscillations could be observed only as EPR correlations provided that the detection of the recoil lepton is related to a neutrino of fixed flavor.

These problems are examined here in a quantum mechanical wave packet treatment of neutrino and neutral meson oscillations which includes the recoil particle in an entangled state. We make explicit certain assumptions concerning correlations between two particles which have space-like separations. An important feature in this approach is that a correlated wave packet can incorporate the effect of both localization and finite lifetime of the source, which is assumed here to be a resonance or unstable particle. It will be shown that these properties explain why the propagation of the particles is confined near classical trajectories. While it has been recognized that classical motion must be combined with wave properties and interference effects for an understanding of the oscillation phenomena, in current discussions classical trajectories have not been introduced in a self-consistent manner [18], [23] [28]. A novel property of our wave packet is that it can incorporate the spacetime coordinates of the decay point of the initial unstable state which can be observed and provides a reference point for the oscillations. Our results averaged over unobserved recoil particle coordinates, Eqs. 28 and 33, differ from those of Dolgov et. al. [18], who assumed that the source and decay particles satisfy exact classical equations motion in violaton of the principles of quantum mechanics.

For simplicity we consider the theory in one dimension where all the 
processes are collinear, and assume there are only two mass eigenstates $|a\rangle$ and $\mid b>$ which is adequate for our purposes. Then the transition amplitude to some final state $\mid g>$ is

$$
A \propto \cos (\theta)<g\left|a>\psi_{a}+\sin (\theta)<g\right| b>\psi_{b}
$$

where $\theta$ is the mixing angle for an initial state of definite flavor or strangeness, and $\psi_{a}$ and $\psi_{b}$ are wavefunctions associated with the different mass eigenstates. We obtain these wavefunctions by time-dependent perturbation theory, assuming that the initial state is a resonance or unstable state of mass $M$ and width $\Gamma$. The wavefunction for this state for $t \geq 0$ is

$$
\psi_{o}(x, t)=\int d p f(p) \exp \left[i p\left(x-x_{s}\right)-\left(i E_{p}+M \Gamma / 2 E_{p}\right)\left(t-t_{s}\right)\right]
$$

where $t_{s}$ is the time at which this state is created as a wave packet centered at $x_{s}, f(p)$ is the amplitude associated with a momentum distribution $p$ in the initial state with corresponding energy $E_{p}=\sqrt{p^{2}+M^{2}}$. In the following discussion we set for convenience $x_{s}, t_{s}$ at the origin of our space-time coordinate system, but it should be remembered that in practice these coordinates are not known precisely and will be included in some of our main results. We assume that this amplitude has a sharp maximum at $p=\bar{p}$, and expanding $E_{p}$ to first order in $p-\bar{p}$ we obtain

$$
\psi_{o}(x, t)=\exp \left[i\left(\bar{p} x-E_{\bar{p}} t\right)\right] \exp \left(-M \Gamma t / 2 E_{\bar{p}}\right) g(x-\bar{v} t)
$$

where the envelope of the wave packet is given directly by the wavefunction of the source at $t=0$

$$
g(x)=\exp (-i \bar{p} x) \psi(x, 0)
$$

In the Wigner-Weisskopf approximation we obtain for $t \geq 0$

$$
\psi_{a, b}\left(x_{1}, x_{2}, t\right)=N \int d p_{1} \int d p_{2} f(p) \frac{\exp \left(i p_{1} x_{1}+i p_{2} x_{2}-i\left(E_{1}+E_{2}\right) t\right)}{\left(E_{1}+E_{2}-E_{p}+i M \Gamma / 2 E_{p}\right)}
$$

where $N=(1 / 2 \pi) \sqrt{\Gamma M\left|\bar{v}_{12}\right| / E_{\bar{p}}}, \bar{v}_{12}$ is the mean relative velocity, and $E_{1}=$ $\sqrt{p_{1}^{2}+m_{1}^{2}}$ and $E_{2}=\sqrt{p_{2}^{2}+m_{2}^{2}}$ are the relativistic energies of the two correlated particles in the final state with masses $m_{1}$ and $m_{2}$ which can have 
different values for the eigenstates labeled $a$ an $b$. Conservation of total momentum in the production process implies that

$$
p=p_{1}+p_{2},
$$

A shortcoming of this representation for $\psi$ is that the state of both particles is given at the same time $t$, while in practice these particles can be detected at different times $t_{1}$ and $t_{2}$. However, since these particles are not interacting, the subsequent time evolution of the wavefunction can be determined by their respective free particle Hamiltonians $H_{1}$ and $H_{2}$. Hence

$$
\psi\left(x_{1}, x_{2}, t_{1}, t_{2}\right)=\exp \left(-i H_{1}\left(t_{1}-t\right)-i H_{2}\left(t_{2}-t\right)\right) \psi\left(x_{1}, x_{2}, t\right),
$$

and the required wavefunction $\psi\left(x_{1}, x_{2}, t_{1}, t_{2}\right)$ (footnote 1 ) is obtained by replacing the factor $\left(E_{1}+E_{2}\right) t$ in Eq. 5 by $E_{1} t_{1}+E_{2} t_{2}$. If these particles are unstable, as is the case for neutral kaons, an additional factor in the integrand of Eq. 5 is required, of the form

$$
\exp -\left(\frac{m_{1} t_{1}}{2 \tau_{1} E_{1}}+\frac{m_{2} t_{2}}{2 \tau_{2} E_{2}}\right)
$$

where $\tau_{1}$ and $\tau_{2}$ are the particle lifetimes. The assumption that the initial state is a resonance or unstable particle of width $\Gamma$ implies an uncertainty in the total energy which plays an essential role in understanding how the decay particles are confined to classical trajectories. Nevertheless, we can define mean momenta $\bar{p}_{1}$ and $\bar{p}_{2}$ associated with the mean total momentum $\bar{p}$ by the requirement that for these special values of momenta, the energy conservation relation

$$
E_{\bar{p}}=\bar{E}_{1}+\bar{E}_{2}
$$

is satisfied exactly.

We carry out the integrations in Eq. 5 approximately, by expanding the momenta $p_{1}$ and $p_{2}$ around these mean values $\bar{p}_{1}$ and $\bar{p}_{2}$, obtained as solutions of the momentum- energy conservation equations, Eqs. 6 and 9 to first order in $p-\bar{p}$ and $E-E_{\bar{p}}$, where $E=E_{1}+E_{2}$. Second order terms contribute to the dispersion of the wave packet which we neglect here. We have

$$
p_{1}=\bar{p}_{1}+\frac{\left(E-E_{\bar{p}}\right)-\bar{v}_{2}(p-\bar{p})}{\bar{v}_{12}},
$$


and

$$
p_{2}=\bar{p}_{2}-\frac{\left(E-E_{\bar{p}}\right)-\bar{v}_{1}(p-\bar{p})}{\bar{v}_{12}},
$$

where $\bar{v}_{1}=\bar{p}_{1} / \bar{E}_{1}, \bar{v}_{2}=\bar{p}_{2} / \bar{E}_{2}$ are the classical or group velocities, and $\bar{v}_{12}=\bar{v}_{1}-\bar{v}_{2}$ is the relative velocity of particles 1 and 2 . Likewise

$$
E_{1}=\bar{E}_{1}+\bar{v}_{1}\left(p_{1}-\bar{p}_{1}\right)
$$

and

$$
E_{2}=\bar{E}_{2}+\bar{v}_{2}\left(p_{2}-\bar{p}_{2}\right)
$$

Changing the coordinates in the integrand of Eq. 5 to the variables $p$ and $E$, we obtain for $t_{12}>0$

$$
\psi\left(x_{1}, x_{2}, t_{1}, t_{2}\right)=N^{\prime} \exp \left[i \phi_{12}\right] \exp \left(-M \Gamma t_{12} / 2 E_{\bar{p}}\right) g\left(z_{12}\right),
$$

where $N^{\prime}=-i \sqrt{\Gamma M / E_{\bar{p}} \bar{v}_{12}}$ is a constant, and

$$
\begin{gathered}
\phi_{12}=\bar{p}_{1} x_{1}+\bar{p}_{2} x_{2}-\bar{E}_{1} t_{1}-\bar{E}_{2} t_{2}, \\
t_{12}=\left(\Delta x_{2}-\Delta x_{1}\right) / \bar{v}_{12}, \\
z_{12}=\left(\bar{E}_{1} \Delta x_{1}+\bar{E}_{2} \Delta x_{2}\right) / E_{\bar{p}},
\end{gathered}
$$

where $\Delta x_{i}=x_{i}-\bar{v}_{i} t_{i}$ for $i=1,2$. For $t_{1}=t_{2}=t$ we have $t_{12}=t-\left(x_{1}-\right.$ $\left.x_{2}\right) / \bar{v}_{12}$ and $z_{12}=x-\bar{v} t$, where $x=\left(\bar{E}_{1} x_{1}+\bar{E}_{2} x_{2}\right) / E_{\bar{p}}$ is the center of mass of the two final state particles. Hence $z_{12}$ is the deviation of the center of mass from classical motion, and $t_{12}=t_{d}$ corresponds to the stochastic time $t_{d}$ at which the particle pair is created at $x_{1}=x_{2}=x_{d}$, where $\phi_{12}=\bar{p} x_{d}-E_{\bar{p}} t_{d}$ and $z_{12}=x_{d}-\bar{v} t_{d}$. At such a point the final state wavefunction is proportional to the initial wavefunction at $x=x_{d}, t=t_{d}$, and it is independent of the mass of the decay particles. Consequently this wavefunction satisfies the initial condition that the flavor of the state be independent of $x_{d}, t_{d}$, an important result which could not be imposed ab initio. As expected, for $t-t_{12}<0$, this wavefunction vanishes.

In our approximation, the initial function, Eq. 2, determines directly the envelope $g\left(z_{12}\right)$ of the wave packet of the two final state particles. Hence the probability for finding these particles at $x_{1}, t_{1}$ and $x_{2}, t_{2}$ is given by

$$
\frac{\Gamma M}{E_{\bar{p}} \bar{v}_{12}} g^{2}\left(z_{12}\right) \exp \left(-\Gamma M t_{12} / E_{\bar{p}}\right) d x_{1} d x_{2}
$$


It can be readily verified that the probability for the creation of these two particles at $x_{d}, t_{d}$ is equal to the probability that the source decays at this same space-time point. If this decay is not measured or constrained by the environment, then Eq. 18 can be applied directly to calculate probabilities or averages over the recoil variable coordinates. However if $t_{d}$ is observed then for times $t_{1} \geq t_{d}$ and $t_{2} \geq t_{d}$ the probability for finding the particles at $x_{1}, x_{2}$ is obtained by setting $t_{12}=t_{d}$ in Eq. 18 (footnote 2), and $x_{1}-x_{2}=$ $\bar{v}_{1}\left(t_{1}-t_{d}\right)-\bar{v}_{2}\left(t_{2}-t_{d}\right)$, or

$$
\Delta_{d} x_{1}=\Delta_{d} x_{2}=z_{12}
$$

where $\Delta_{d} x_{i}=x_{i}-\bar{v} t_{d}-\bar{v}_{i}\left(t_{i}-t_{d}\right)$ is the deviation from classical motion of the decay particles. In this case, the measure $z_{12}$ for this deviation satisfies he same distribution as the deviation from classical motion of the initial state, and therefore our analysis shows that particles associated with the decay process are confined to move along classical trajectories with the same degree of localization as the source.

We now assume that the two states labeled $a$ and $b$ correspond to particles 1 and 2 with small mass differences, $\delta m_{i}^{2}=m_{i a}^{2}-m_{i b}^{2}$, and calculate the corresponding differences in the mean momentum $\bar{p}_{1}$ and $\bar{p}_{2}$ and corresponding energies $\bar{E}_{1}$ and $\bar{E}_{2}$ from the energy-momentum conservation laws, Eqs. 6 and 9. We have

$$
\delta \bar{p}_{1}+\delta \bar{p}_{2}=0
$$

and

$$
\delta \bar{E}_{1}+\delta \bar{E}_{2}=0
$$

where to first order in $\delta m_{i}^{2}$,

$$
\delta \bar{E}_{i}=\bar{v}_{i} \delta \bar{p}_{i}+\frac{\delta m_{i}^{2}}{2 \bar{E}_{i}} .
$$

Solving these equations we obtain

$$
\delta \bar{p}_{1}=-\delta \bar{p}_{2}=-\frac{1}{\bar{v}_{12}}\left(\frac{\delta m_{1}^{2}}{2 \bar{E}_{1}}+\frac{\delta m_{2}^{2}}{2 \bar{E}_{2}}\right) .
$$

where $\bar{v}_{12}=\bar{v}_{1}-\bar{v}_{2}$ is the relative velocity. These relations differ from the result obtained with conventional kinematics assumptions that different mass states have either the same momentum [6] - 8] or the same energy [1] - [14]. 
The oscillation term which concerns us here appears in the calculation of the interference term in the transition probability $|A|^{2}$, where $A$ is given by Eq. 1, and is proportional to

$$
\operatorname{Real} \psi_{a}^{*} \psi_{b}=\cos (\phi) g\left(z_{12, a}\right) g\left(z_{12, b}\right) \exp \left(-M \Gamma\left(t_{12, a}+t_{12, b}\right) / 2 E_{\bar{p}}\right)
$$

where we have ignored factors which depend on the lifetime of the final state particles, Eq. 8. The phase difference $\phi=\phi_{12, a}-\phi_{12, b}=\delta \bar{p}_{1} x_{1}+\delta \bar{p}_{2} x_{2}-$ $\delta \bar{E}_{1} t_{1}-\delta \bar{E}_{2} t_{2}$ is invariant under Lorentz transformations, and according to the energy-momentum conservation laws, Eqs. 20 and 21, it can be written in the form

$$
\phi=\delta \bar{p}_{1}\left(x_{1}-x_{2}\right)-\delta \bar{E}_{1}\left(t_{1}-t_{2}\right) .
$$

This form shows that the phase difference $\phi$ depends only on the relative time coordinate of the final state particles and, therefore, that it is independent of the initial decay point $t_{d}$. However the role of this decay time appears when we consider the effect of the wave packet envelope in the case that this coordinate is measured, constraining each of the decay particles to move near its classical trajectories. For this purpose we apply Eq. 22 to write $\phi$ in the equivalent form

$$
\phi=\delta \bar{p}_{1}\left(\Delta x_{1}-\Delta x_{2}\right)-\frac{\delta m_{1}^{2}}{2 \bar{E}_{1}} t_{1}-\frac{\delta m_{2}^{2}}{2 \bar{E}_{2}} t_{2},
$$

This expression for $\phi$ is similar to results given in [18] and [28], but our correlated wave packet now allows us to interpret and evaluate properly the contribution of the first term in $\phi$ which does not appear in the convential single particle formulation for this phase. Substituting Eq. 23 for $\delta \bar{p}_{1}$ and substituting $\Delta x_{2}-\Delta x_{1}=\bar{v}_{12} t_{12}$, we obtain finally

$$
\phi=-\frac{\delta m_{1}^{2}}{2 \bar{E}_{1}}\left(t_{1}-t_{12}\right)-\frac{\delta m_{2}^{2}}{2 \bar{E}_{2}}\left(t_{2}-t_{12}\right),
$$

If the recoil particle has a fixed mass, i.e. $\delta m_{2}^{2}=0$, this form for $\phi$ is equal to the conventional single particle result with mass eigenstates of the same momentum provided that we identify $t_{12}$ with the decay time $t_{d}$ of the initial state as we have done previously, see footnote 2 . In this case this phase is independent of the recoil particle coordinates contrary to assertions in [23]-[26]. However, if the decay time $t_{d}$ is not measured directly, then in 
principle it could be determined from a coincidence measurement of the recoil coordinate $x_{2}$. If we set $t_{1}=t_{2}$, and substitute $t_{1}-t_{12}=\left(x_{1}-x_{2}\right) / \bar{v}_{12}$ in Eq. 27, we obtain EPR-like oscillations in the relative coordinates of the two final state particles which can only be observed if the flavor is also determined [18]. In the case of neutral meson pairs in the final state, e. g. $K \bar{K}$ produced in $\phi$ decay, $\delta m_{2}^{2}=-\delta m_{1}^{2}$, and the time coordinates of both particles appear in Eq. 27 even if the decay time $t_{d}$ has been determined. A similar result was obtained in 21] and 22 by assuming that $\delta \bar{p}_{1}=\delta \bar{p}_{2}=0$, although this kinematical condition is not justified.

If neither the decay time $t_{d}$ nor the coordinates $x_{2}, t_{2}$ of the recoil particle are observed, we must integrate the interference term of the transition probability, Eq. 24 over the decay time $t_{d}$, or what amounts to an equivalent procedure, over the position coordinate $x_{2}$ of the unobserved recoil particle. Assuming that there are no enviromental constraints on the possible range of these variable, we apply eq. 18 to obtain the average of $\cos (\phi)$. Neglecting the mass difference in the envelopes of the wave packet, which leads to a finite coherence length, we obtain

$$
<\cos (\phi)>=R \cos \left(\frac{\delta m_{1}^{2}}{2 \bar{E}_{1}}\left(t_{1}-t_{s}\right)+\frac{\delta m_{2}^{2}}{2 \bar{E}_{2}}\left(t_{2}-t_{s}\right)-\delta\right),
$$

where

$$
\begin{gathered}
R=\frac{1}{\sqrt{1+\xi^{2}}}, \\
\tan (\delta)=\xi,
\end{gathered}
$$

and

$$
\xi=\left(\frac{\delta m_{1}^{2}}{2 \bar{E}_{1}}+\frac{\delta m_{2}^{2}}{2 \bar{E}_{2}}\right)\left(\frac{\bar{E}_{p}}{M \Gamma}\right)
$$

This average is independent of the shape of the initial wave packet. The parameter $\xi$ gives a measure for the deviation from the conventional form, which corresponds to $R=1$ and $\delta=0$ in Eq. 28. For example, in particle reactions producing neutral mesons, $\Gamma$ is of order several $M e V$, and for the $\mathrm{B}$ meson $\delta m$ is $3.1 \times 10^{-4} \mathrm{eV}$, and about 100 times smaller for the $\mathrm{K}$ meson. Hence $\xi$ is of order $10^{-10}-10^{-12}$, and the contribution from the first term which appears in Eq. 26 is essentially unobservable, contrary to expectations in [28]. For neutrinos produced in pion decay, with $\delta m^{2} \approx 10^{-3} \mathrm{eV}^{2}$ as found in [5], $\xi \approx 10^{-3}$ can also be neglected in the analysis of the data. However, 
this is not the case for $\delta m^{2}$ of order $1-10 \mathrm{eV}^{2}$ as reported in [ [ 1 ]. Furthermore, the magnitude of $\xi$ is greater for the case that the neutrinos are produced in muon decay [3] because the muon is lighter than the pion and has a longer life time, but this is a three body decay and our analysis is only approximatly valid.

In practice it must be remembered that oscillations are observed in position rather than in time coordinates. Setting $t_{i}=\left(x_{i}-\Delta x_{i}\right) / \bar{v}_{i}$ in Eq. 27 for the phase we obtain an equivalent form

$$
\phi=\left(\frac{\delta m_{1}^{2}}{2 p_{1}}+\frac{\delta m_{2}^{2}}{2 p_{2}}\right) z_{12}-\frac{\delta m_{1}^{2}}{2 \bar{p}_{1}}\left(x_{1}-\bar{v} t_{12}\right)-\frac{\delta m_{2}^{2}}{2 \bar{p}_{2}}\left(x_{2}-\bar{v} t_{12}\right) .
$$

This differs from the conventional form for $\phi$, but if the decay coordinates are determined then $t_{12}=t_{d}, z_{12}=\Delta_{d} x_{1}=\Delta_{d} x_{2}$, Eq. 19, and we recover the conventional form. Notice that in the rest frame of the source $\bar{v}=0$, and the dependence of the phase on $t_{12}$ vanishes because the source is not moving. Assuming a Gaussian distribution for the initial wave packet with a width $\sigma_{x}$, and averaging $\cos (\phi)$ over $z_{12}$ and $t_{12}$ we now obtain

$$
<\cos (\phi)>=\bar{R} \cos \left(\frac{\delta m_{1}^{2}}{2 \bar{p}_{1}}\left(x_{1}-x_{s}\right)+\frac{\delta m_{2}^{2}}{2 \bar{p}_{2}}\left(x_{2}-x_{s}\right)-\bar{\delta}\right),
$$

where

$$
\begin{gathered}
\bar{R}=\frac{\exp \left(-\eta^{2}\right)}{\sqrt{1+\bar{\xi}^{2}}}, \\
\tan (\bar{\delta})=\bar{\xi} \\
\bar{\xi}=\left(\frac{\delta m_{1}^{2}}{2 \bar{p}_{1}}+\frac{\delta m_{2}^{2}}{2 \bar{p}_{2}}\right)\left(\frac{\bar{p}}{M \Gamma}\right),
\end{gathered}
$$

and

$$
\bar{\eta}=\left(\frac{\delta m_{1}^{2}}{2 \bar{p}_{1}}+\frac{\delta m_{2}^{2}}{2 \bar{p}_{2}}\right) \sigma_{x}
$$

As expected from simple physical arguments, interference effects can occur in position measurements provided that the width $\sigma_{x}$ of the wave packet is small compared to the oscillation length $\propto \bar{p}_{i} / \delta m_{i}^{2}$, or correspondingly that $\eta \propto \delta \bar{p}_{i} \sigma_{x}<<1$. The magnitude of $\sigma_{x}$ is of the order of magnitude of the localization of a nuclear target, and it is further contracted by the Lorentz transformation due to the motion of the unstable initial particle so that in 
practice $\eta<<1$. Moreover, in the rest frame of the source $\bar{p}=0$ and consequently in this case $\bar{\xi}=0$.

In conclusion, we have shown that the transition probability for neutrino and neutral meson oscillations can be obtained from first principles by solving the time dependent Schrödinger equation for the decay of an unstable source into a coherent superposition of correlated two particle eigenstates with different masses. We have obtain our results in a relativistically covariant manner by applying well defined approximations without recourse to conventional ad-hoc assumptions which violate principles of quantum mechanics, and have led to much confusion in the literature. We have shown that the width or lifetime of the source plays a crucial role in understanding this problem, and that quantum correlations between the final state particles relate the decay time of the source to the space-time coordinates of the these particles. Due to the mass difference the wave packets for different mass eigenstates have different group velocities and separate leading to a finite coherence length [15], but this effect was neglected here.

\section{Acknowledgements}

I would like to thank D. Dorfan and A. Seiden for very stimulating discussions, critical questions, and a careful reading of this manuscript, and $\mathrm{T}$. Goldman and Ann Nelson for several useful comments.

\section{Footnotes}

1. The justification for Eq. 7 is that after one of the two particles has been detected, i.e. $t=t_{1}$ or $t=t_{2}$, its state does not continue to evolve in time. The generalized wavefunction $\psi\left(x_{1}, x_{2}, t_{1}, t_{2}\right)$, Eq. 14 can then be interpreted as the probability amplitude for correlated events which occur at the two different space-time points $\left(x_{1}, t_{1}\right)$ and $\left(x_{2}, t_{2}\right)$, and is equivalent to the amplitude method in [21], and the formalism in [18]. It can be shown that this procedure is equivalent to the "collapse" of the wavefunction language, which is the conventional description when measurements take place at different times, although it is preferable not to invoke this awkward language.

2. For $t_{1}, t_{2} \geq t_{d}$, the condition $t_{12}=\left(\bar{v}_{1} t_{1}-\bar{v}_{2} t_{2}-x_{1}+x_{2}\right) / \bar{v}_{12}=t_{d}$ 
connects the decay time $t_{d}$ of the source to the space-time coordinates of the decay particles. It corresponds to the classical relation for the relative coordinates of these particles which can be understood on the physical grounds that these particles are created in a region of negligible small spatial dimension, without violating the uncertainty principle because $t_{d}$ is a stochastic variable. This relation has also been obtained in [18, but under the invalid assumption that the source and decay particles follow classical trajectories exactly. In the case that the decay can occur over a range of values $0 \leq t_{d} \leq t_{\max }$, one must take an average over the probability distribution integrated over this range provide there are no measurements on the recoil particle which constrain the possible values of $t_{12}$. In practice $t_{\max }=d / \bar{v}$, where $d$ is the distance between the target where the unstable particle is created, and a beam stop where nuclear reactions annihilate it.

\section{References}

[1] J. Bell and J. Steinberger, Proceedings Oxford International Conference on Elementary Particles 1965, and Quantum mechanics, high energy physics and accelerators ed. M. Bell, (CERN, 1996) 280-307

[2] F. Abe, et. al. Phys. Rev. Lett. 80 (1998) 2057

[3] C. Athanassopoulous et al., Phys. Rev. C 54 (1996) 2685; Phys. Rev. Lett. 77 (1996) 3082

[4] C. Athanassopoulous et al., Phys. Rev. Lett. 81 (1998) 1774

[5] Y. Fukuda et al., Phys. Rev. Lett. 81 (1998) 1562

[6] S. Nussinov, Phys. Lett. B 63 (1976) 201

[7] S. M. Bilenky and B. Pontecorvo, Phys. Rep. 41 (1978) 25

[8] B. Kayser, Phys. Rev. D 24 (1981) 110

[9] Felix Boehm and Peter Vogel, Physics of Massive Neutrinos (Cambridge University Press, 1992) 
[10] C. W. Kim and A. Pevsner, Neutrinos in Physics and Astrophysics (Harwood Academic, 1993)

[11] H. J. Lipkin, Phys. Lett. B 348(1995) 604

[12] L. Stodolsky, Phys. Rev. D 58 (1998) 036006

[13] Y. Grossman and H. J. Lipkin, Phys. Rev. D 55 (1997)2760

[14] H.J. Lipkin, Quantum Mechanics of Neutrino Oscillations - Hand Waving for Pedestrians 1998 Neutrino Oscillation Workshop , Amsterdam (to be published)

[15] C. Giunti, C.W. Kim and U.W. Lee, Phys. Rev. D44 (1991) 3635; Phys. Rev. D 45 (1992) 2414

[16] J. Rich, Phys. Rev. D 48 (1993) 4318

[17] T. Goldman, Source Dependence of Neutrino Oscillations hepph/9604357

[18] A.D. Dolgov, A.Yu. Morozov, L.B. Okun, and M.G. Schepkink, Nucl. Phys. 502 (1997) 3

[19] A. Einstein, B. Podolsky and N. Rosen, Phys. Rev. 47 (1935) 777

[20] H. J. Lipkin, Phys. Rev. 176 (1968) 1715

[21] B. Kayser and L. Stodolsky, Phys. Lett. B 359 (1995) 343

[22] I. Dunietz, J Hauser and J. I. Rosner, Phys. Rev. D 35 (1987) 2166

[23] Y. N Srivastava, A. Widom and E. Sassaroli, Phys. Lett. B 334 (1995) 436

[24] Y. N Srivastava, A. Widom and E. Sassaroli, The European Physical Journal C 2, 769-774 (1998)

[25] A. Widom Y.N. Srivastava, Lambda Oscillations and the Conservation Laws hep-ph/96045399 
[26] Y. N. Srivastawa and A. Widom, Of Course Muons Can Oscillate hep$\mathrm{ph} / 970726$

[27] J. Lowe, B. Bassalleck, H. Burkhardt, A Rusek, G. J. Stephenson Jr. and T. Goldman, Phys. Lett.B 384, 288 (1996)

[28] H. Burkhard, J. Lowe, G.J. Stephenson Jr. and T. Goldman, Oscillations of recoil particles against mixed states hep-ph/9803365 